\title{
Bringing Simulation to Life: A Mixed Reality Autonomous Intersection
}

\author{
Michael Quinlan, Tsz-Chiu Au, Jesse Zhu, Nicolae Stiurca, Peter Stone
}

\begin{abstract}
Fully autonomous vehicles are technologically feasible with the current generation of hardware, as demonstrated by recent robot car competitions. Dresner and Stone proposed a new intersection control protocol called Autonomous Intersection Management (AIM) and showed that with autonomous vehicles it is possible to make intersection control much more efficient than the traditional control mechanisms such as traffic signals and stop signs. The protocol, however, has only been tested in simulation and has not been evaluated with real autonomous vehicles. To realistically test the protocol, we implemented a mixed reality platform on which an autonomous vehicle can interact with multiple virtual vehicles in a simulation at a real intersection in real time. From this platform we validated realistic parameters for our autonomous vehicle to safely traverse an intersection in AIM. We present several techniques to improve efficiency and show that the AIM protocol can still outperform traffic signals and stop signs even if the cars are not as precisely controllable as has been assumed in previous studies.
\end{abstract}

\section{INTRODUCTION}

Intelligent Transportation Systems (ITS) is a multidisciplinary field concerned with advancing modern transportation systems with information technology [1]. Most existing work on ITS focusses on assisting human drivers to avoid collision in intersections [2], [3]. But the DARPA Urban Challenge in 2007 showed that fully autonomous vehicles are technologically feasible [4]. We therefore believe that the time is right to rethink our current transportation infrastructure, which is designed solely for human drivers.

To this end, Dresner and Stone proposed a multiagent systems approach to autonomous intersection management, and in particular described a First Come, First Served (FCFS) policy for directing vehicles through an intersection [5]. This approach has been shown, in simulation, to yield significant improvements in intersection performance over conventional intersection control mechanisms such as traffic signals and stop signs. Despite, or perhaps because of the impressive performance, questions are often raised regarding its applicability to real autonomous vehicles. In this paper we take the first step to show that a real autonomous vehicle can adhere to the FCFS protocol and efficiently traverse an intersection.

Fully testing the protocol requires a fleet of autonomous vehicles, which is too costly and is potentially unsafe given the risk of collisions during testing. In this paper, we present a mixed reality platform on which a single autonomous vehicle can interact with virtual traffic in a simulation. This platform can provide a high degree of realism for testing the

Department of Computer Science, The University of Texas at Austin. \{mquinlan, chiu, jzhu, nstiurca, pstone\}@cs.utexas.edu performance of real autonomous vehicles at real intersections under the FCFS policy.

Based on numerous tests on our mixed reality platform we obtained more accurate details regarding the parameters the intersection controller should use to guarantee safe traversal of real autonomous vehicles in real intersections. In particular we confirmed that the noise and imprecision of a real vehicle is greater than that anticipated in previous simulations. To account for these deficiencies, FCFS must enlarge the buffers surrounding vehicles, in effect pretending that the vehicles are larger than they really are, thus providing some degree of slack to account for potential imprecisions in control. However, this use of large buffer sizes can lead to significant inefficiencies in FCFS. In this paper, we use Little's Law to explicate how the performance of an autonomous vehicle relates to the overall intersection throughput, and identify approaches to increase the throughput in order to compensate for the deficiencies due to the enlarged buffers. Our experiments show that even with increased buffers, FCFS can still provide substantial performance improvements over traffic signals and four-way stop signs.

\section{AUTONOMOUS INTERSECTION MANAGEMENT}

The novel intersection management introduced by Dresner and Stone is based on a reservation paradigm, in which vehicles "call ahead" to reserve space-time in the intersection [5]. They assume that computer programs called driver agents control the vehicles, while an arbiter agent called an intersection manager is placed at each intersection. The driver agents attempt to reserve a block of space-time in the intersection. The intersection manager decides whether to grant or reject requested reservations according to an intersection control policy. In brief, the paradigm proceeds as follows.

- An approaching vehicle announces its impending arrival to the intersection manager. The vehicle indicates its size, predicted arrival time, velocity, acceleration, and arrival and departure lanes.

- The intersection manager simulates the vehicle's path through the intersection, checking for conflicts with the paths of any previously processed vehicles.

- If there are no conflicts, the intersection manager issues a reservation. It then becomes the vehicle's responsibility to arrive at, and travel through, the intersection as specified.

- The car may only enter the intersection once it has successfully obtained a reservation.

Fig. 1 diagrams the interaction between driver agents and an intersection manager. A key feature of this paradigm is 
that it relies only on vehicle-to-infrastructure (V2I) communication. ${ }^{1}$ In particular, the vehicles need not know anything about each other beyond what is needed for local autonomous control (e.g., to avoid running into the car in front). The paradigm is also completely robust to communication disruptions: if a message is dropped, either by the intersection manager or by the vehicle, delays may increase, but safety is not compromised. Safety can also be guaranteed in scenarios when both autonomous and human driven vehicles operate at intersections. The intersection efficiency increases with the ratio of autonomous vehicles to human driven vehicles in such scenarios.

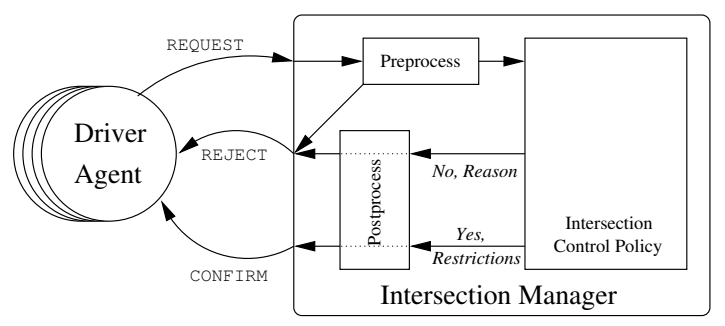

Fig. 1. Diagram of the intersection system.

The prototype intersection control policy divides the intersection into a grid of reservation tiles, as shown in Fig. 2. When a vehicle approaches the intersection, the intersection manager uses the data in the reservation request regarding the time and velocity of arrival, vehicle size, etc. to simulate the intended journey across the intersection. At this stage the intersection manager also introduces safety buffers that further ensure a safe traversal of the intersection. AIM currently consists of three buffer types: 1) the Static Buffer represents a constant sized buffer around the vehicle, for example $0.5 \mathrm{~m}$ for GPS error; 2) the Internal Time Buffer adds a buffer in the direction of travel that extends for $x$ seconds, thus allowing a vehicle to arrive $x$ seconds early or late; and 3) the Edge Time Buffer creates a gap of $x$ seconds at the edge of the intersection, thus preventing vehicles from exiting too close to the previous vehicle. At each simulated time step, the policy determines which reservation tiles will be occupied by the vehicle (including safety buffers).

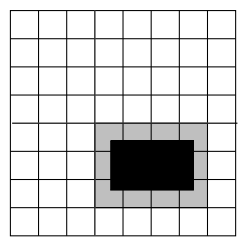

(a) Successful

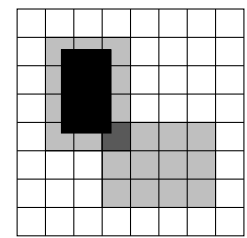

(b) Rejected
Fig. 2. (a) The vehicle's space-time request has no conflicts at time $t$. (b) The black vehicle's request is rejected because at time $t$ of its simulated trajectory, the vehicle requires a tile already reserved by another vehicle. The shaded area represents the static buffer of the vehicle.

\footnotetext{
${ }^{1} \mathrm{~A}$ V2V version with no infrastructure has been developed for low-traffic intersections [5].
}

If at any time during the trajectory simulation the requesting vehicle occupies a reservation tile that is already reserved by another vehicle, the policy rejects the driver's reservation request, and the intersection manager communicates this to the driver agent. Otherwise, the policy accepts the reservation and reserves the appropriate tiles. The intersection manager then sends a confirmation to the driver. If the reservation is denied, it is the vehicle's responsibility to maintain a speed such that it can stop before the intersection. Meanwhile, it can request a different reservation.

Empirical results in simulation demonstrate that the proposed reservation system can dramatically improve the intersection efficiency when compared to traditional intersection control mechanisms. To quantify efficiency, Dresner and Stone introduce delay, defined as the amount of travel time incurred by the vehicle as the result of passing through the intersection. According to their experiments, the reservation system performs very well, nearly matching the performance of the optimal policy which represents a lower bound on delay should there be no other cars on the road (Fig. 14 in [5]). Overall, by allowing for much finer-grained coordination, the simulation-based reservation system can dramatically reduce per-car delay by two orders of magnitude in comparison to traffic signals and stop signs.

\section{REALITY CHECK: AIM IN PRACTICE}

Simulations inevitably approximate critical aspects of reality. Real vehicles may not be as precisely controllable as the vehicles in simulations, nor may GPS provide as reliable location estimates. In this section, we introduce the concept of mixed reality simulation that enables the testing of a single real autonomous vehicle as it interacts with many virtual ones. Thus we dramatically reduce the cost and risk of testing, while still grounding the system in reality.

\section{A. Mixed Reality Platform}

Our mixed reality platform is built on top of Marvin-our autonomous vehicle [6] (Fig. 3(a)) - and the AIM simulator (Fig. 3(b)). The key aspect is the addition of proxy vehicles to the AIM software. Each proxy vehicle acts as a gateway between a real autonomous vehicle, such as Marvin, and the simulated environment.

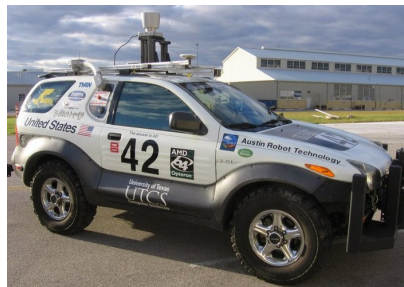

(a) Marvin

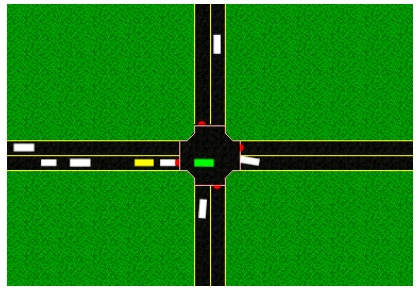

(b) The AIM simulator
Fig. 3. The full sized autonomous vehicle and the AIM simulator in our mixed reality platform. The green rectangle in the middle of the intersection is a proxy vehicle representing the real world location of the autonomous vehicle. The white rectangles are virtual vehicles with reservations, while the yellow rectangles are virtual vehicles without reservations. 
From the viewpoint of the intersection manager, the proxy vehicle is identical to other virtual vehicles; the driver agent on Marvin communicates with the intersection manager by sending and receiving AIM messages via UDP. The motion of the proxy vehicle, however, is controlled by Marvin, which regularly updates the location, the direction, and the velocity of the proxy vehicle via UDP packets containing the realtime GPS information of the actual vehicle.

In our setup, we ran the AIM simulator adjacent to the driver agent of Marvin on a computer located in Marvin. At the beginning, the simulator generated potentially more than 100 virtual vehicles to create steady traffic in the simulated world. Then the driver agent connected to the simulator and the simulator created a proxy vehicle whose physical state (the position, the direction, etc) was the same as Marvin's. As described above, the proxy vehicle interacted with other virtual vehicles on behalf of Marvin. As Marvin approached an intersection (and thus so did the proxy vehicle on the simulator's map), the driver agent sent a request message over a specified UDP port to the proxy vehicle, which then forwarded the message to the intersection manager in the simulator. The intersection manager either accepted or rejected the reservation request based on the virtual traffic at the intersection. Once a reservation was granted, the proxy vehicle forwarded the confirm message to Marvin, and Marvin progressed through the intersection in both the realworld and the simulated world accordingly. An example of this experiment can be seen in the accompanying video.

\section{B. Adjusting Simulation Parameters}

The purpose of the mixed reality platform is to 1) test the performance of AIM with real autonomous vehicles in real world settings, and 2) identify aspects of the simulation that failed to capture the true behavior of the real autonomous vehicle. In this section, we present our findings and discuss how we adjusted the simulation parameters to compensate for the modeling errors for safe traversal in intersections.

Our tests were undertaken on a road with a legal speed limit of $20 \mathrm{mph}(8.94 \mathrm{~m} / \mathrm{s})$, for the safety of others the maximum speed (Max Speed) of both Marvin and the virtual vehicles were set to $7.5 \mathrm{~m} / \mathrm{s}$. The AIM simulator requires us to define the operating characteristics of Marvin such as its size and its maximum acceleration in order to compute its trajectory in the intersection. Although Marvin is technically capable of accelerating at $2.5 \mathrm{~m} / \mathrm{s}^{2}$, the acceleration in practice rarely exceeds $0.5 \mathrm{~m} / \mathrm{s}^{2}$ due to driver discomfort and the controller's safety measures. Similarly, Marvin may drift marginally below the speed limit for safety reasons and to facilitate smoother braking when approaching a stop.

Apart from the operating characteristics of Marvin, we need to choose the values for three additional parameters: Static Buffer Size, Internal Time Buffer and Edge Time Buffer. These parameters are used in AIM to factor in the GPS errors and the vehicle controller's errors due to sensor noise and road conditions. We find that the default values of these parameters $(0.25,0.0$ and 0.5 , respectively) in the simulator are too small for Marvin since the errors in the real world are much larger than the errors in simulation. Therefore, we needed to figure out how large the buffer sizes should be such that Marvin can stay inside the buffer during its entire traversal. After a series of empirical test, we settled on the following numbers: Static Buffer Size $=1 \mathrm{~m}$, Internal Time Buffer $=2$ seconds and Edge Time Buffer $=4$ seconds.

To better understand the impact of the parameter modifications, we ran simulations to measure the average delay across a range of traffic densities. In Fig. 4, the green dots (2nd line from the bottom) are the data points for the default parameters: Max Speed $=25 \mathrm{~m} / \mathrm{s}$, Max Acceleration $=4 \mathrm{~m} / \mathrm{s}^{2}$, Static Buffer Size $=0.25 \mathrm{~m}$, Internal Time Buffer $=0 \mathrm{~s}$, and Edge Time Buffer $=0.5 \mathrm{~s}$. The red dots (highest line) are the data points for the modified parameters: Max Speed $=7.5 \mathrm{~m} / \mathrm{s}$, Max Acceleration $=0.5 \mathrm{~m} / \mathrm{s}^{2}$, Static Buffer Size $=1 \mathrm{~m}$, Internal Time Buffer $=2 \mathrm{~s}$, and Edge Time Buffer $=4 s$. As expected, either having a more conservative estimation of vehicles' speed limit and maximum acceleration or increasing any buffer sizes indeed increase the average delay of a vehicle. When the traffic rate is larger than 200 vehicles per hour per lane, traffic congestion occurs and the averaoe delav increases tremendnuclv

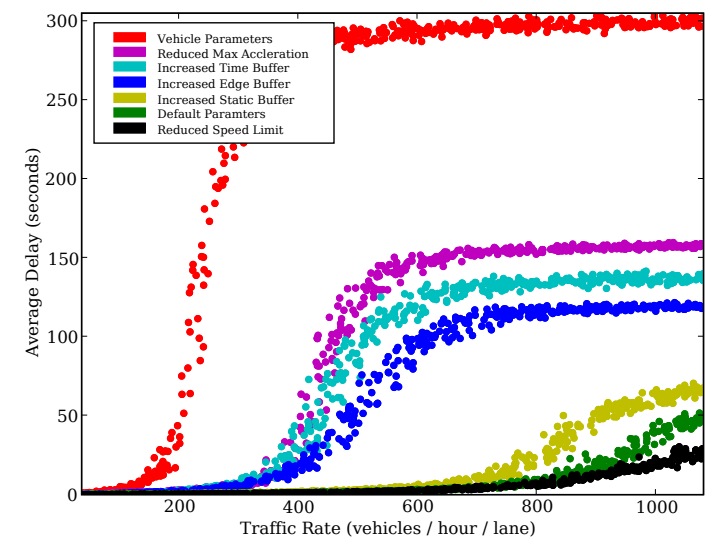

Fig. 4. The effect of modifying simulation parameters. The green dots (2nd line from bottom) are the data points collected using the default simulation parameters that are used in previous studies. The red data points (top line) are generated using the parameters required by Marvin to safely traverse the intersection according to our empirical test in the mixed reality platform. The other data points demonstrate the effect of substituting the default parameters with the required parameters one at a time. For example, 'Reduced Max Acceleration' (2nd line from top) uses all parameters in previous studies except Max Acceleration, which is changed from $4.0 \mathrm{~m} / \mathrm{s}^{2}$ to $0.5 \mathrm{~m} / \mathrm{s}^{2}$.

\section{COMPENSATING FOR LARGE BUFFERS}

The primary approach for coping with any noise present in the vehicle's system that can cause a significant level of uncertainty and result in position and velocity errors is to increase the buffer sizes so that vehicles do not collide even when they deviate from their expected paths. However, increasing the buffer sizes also decreases the throughput of the intersection, and may cause traffic congestion. In this section we present two techniques to increase the throughput of an intersection and compensate for the effects of large buffer sizes. 


\section{A. Static Buffer Sizes vs. Throughput}

To understand how the buffer size affects the maximum throughput characteristics of intersections, we borrow some tools from queueing theory. An important result in queueing theory is Little's Law [7], which states that in a queueing system the average arrival rate of customers, $\lambda$, is equal to the average number of customers, $T$, in the system divided by the average time, $W$, a customer spends in the system. In the context of intersection management, Little's Law can be written as $L=\lambda W$, where

- $L$ is the average number of vehicles in the intersection;

- $\lambda$ is the average arrival rate of the vehicles at the intersection; and

- $W$ is the average time a vehicle spends in the intersection. Note that the arrival rate is equal to the throughput of the system since no vehicle stalls inside an intersection.

Little's Law shows that the maximum throughput (i.e., the upper bound of $\lambda$ ) an intersection can sustain is equal to the upper bound of $L$ divided by the lower bound of $W$, where the upper bound of $L$ is the maximum number of vehicles that can coexist in an intersection, and the lower bound of $W$ is the minimum time a vehicle spends in the intersection. Thus, Little's Law shows that there are two ways to increase the maximum throughput: 1) increase the average number of vehicles in an intersection at any moment of time, and 2) decrease the average time a vehicle spends in an intersection.

A trivial upper bound on $L$ is the area of the intersection divided by the average static buffer size of the vehicles. But this bound is rather loose and in practice unachievable. Nonetheless, it provides us some hints about the dependence between the maximum throughput and the average static buffer size of the vehicles. In general, an increase in static buffer size has two effects: 1) the maximum number of vehicles in each trajectory will decrease; and 2) the number of nonintersecting trajectories decreases, since a large static buffer effectively increases the width of the trajectories, causing trajectories to overlap one another. In both cases, the average number of vehicles decreases as the static buffer size increases. According to Little's Law, this can reduce the throughput of an intersection. To see whether it is the case, we ran an experiment using our traffic simulator with an implementation of FCFS. In the experiment, we set the traffic level of each lane to 576 vehicles per hour, the speed limit of the roads to $25 \mathrm{~m} / \mathrm{s}$, the maximum acceleration of the vehicles to $4 \mathrm{~m} / \mathrm{s}^{2}$, the internal time buffer to zero seconds and the edge time buffer to $0.25 \mathrm{~s}$. We then varied the size of the static buffers between 0 and 6 meters to observe how it affects the average delay and the throughput. Fig. 5 shows the result of these experiments. From this figure, we can see that the average delay of a vehicle does increase as the static buffer size increases. Interestingly, when the static buffer size is larger than 2 meters, the average delay suddenly increases by a factor of two. This transition point is due to the fact that a static buffer larger than 2 meters causes the width of trajectories to be larger than the width of a lane, and this reduces the number of the nonintersecting trajectories by approximately half. In general, the average delay increases linearly with the static buffer size, except at this transition point. The throughput (i.e., the arrival rate of the vehicles at the intersection), however, decreases moderately, because most of the vehicles eventually enter the intersection even though they have longer delays.
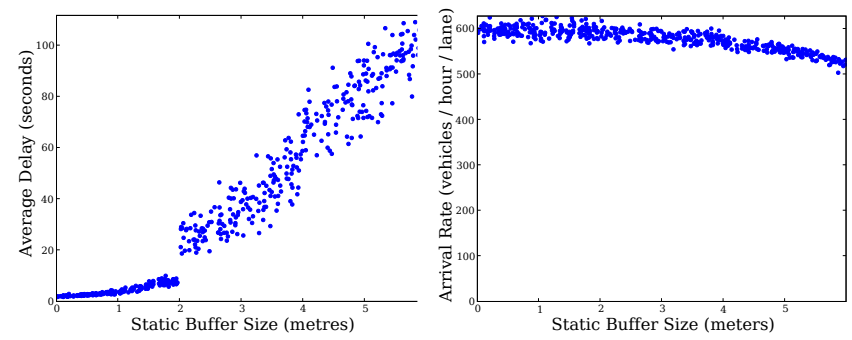

Fig. 5. Static buffer size versus average delay and arrival rate.

\section{B. Reducing Traversal Time by Increasing Acceleration}

We have shown that the static buffer size has a great impact on the average delay and the throughput of an intersection. But the size of an intersection is a hard limit and the static buffer sizes must reflect the vehicle's precision of controlthere is little an intersection manager can do to squeeze more vehicles into the intersection.

Fortunately, Little's Law shows that there is another way to increase the throughput-reducing the average time a vehicle takes to traverse an intersection. In other words, a vehicle should maintain a high speed during the traversal of an intersection in order to shorten its traversal time. A vehicle's velocity in an intersection depends on two factors: 1) the initial velocity when the vehicle enters the intersection, and 2) the acceleration during the traversal. In this section, we will examine how the acceleration of vehicles in an intersection can affect the intersection's throughput. In Section IV-C, we will present a technique to prevent vehicles from stopping before the intersection such that vehicles can enter the intersection at a higher initial velocity.

We conducted an experiment using our simulator to see the change in average delay and throughput as the maximum acceleration of the vehicle increases. In the experiment, we again set the traffic level of each lane to 576 vehicles per hour, the speed limit of the roads to $25 \mathrm{~m} / \mathrm{s}$, the static buffer size to $0.25 \mathrm{~m}$, the internal time buffer to zero seconds and the edge time buffer to $0.25 \mathrm{~s}$. The maximum acceleration of the vehicles is then varied from 0 to $6 \mathrm{~m} / \mathrm{s}^{2}$, with the results shown in Fig. 6. We can see that as the maximum acceleration increases the average delay drops to zero quickly and the throughput increases to a level that is identical to the incoming traffic level. To explain why it is the case, let us consider the relationship between the lower bound of the traversal time and the maximum acceleration. Let $v_{0}$ be the arrival (initial) velocity of the vehicle when it enters an intersection, $d$ be the length of its trajectory in the intersection, $v_{1}$ be the velocity when the vehicle leaves the intersection, $W$ be the time the vehicle spends in the intersection, and $a$ be the acceleration, assuming that 
the vehicle accelerates at a constant rate during the entire traversal. If $v_{0}$ is almost equal to the maximum velocity of the vehicle and the speed limit of the road, the acceleration has little effect on the traversal time $W$; otherwise, we have $W=\frac{\sqrt{v_{0}^{2}+2 a d}-v_{0}}{a}$ by solving the following two equations: $v_{1}=v_{0}+a W$ and $d=W\left(v_{1}+v_{0}\right) / 2$. If $v_{0}$ is 0 , then $W=\frac{\sqrt{2 d}}{\sqrt{a}}$, and the throughput $\lambda$, which is inversely proportional to $W$, is proportional to $\sqrt{a}$. If $v_{0}$ is not zero, we can check that $\lambda$ is approximately proportional to $\sqrt{a}$. This explains the shape of the line in Fig. 6(b). If the traffic level is smaller than the maximum throughput an intersection can sustain, almost all vehicles can pass through the intersection without stopping, and this is indeed the case when the maximum acceleration is larger than $2 \mathrm{~m} / \mathrm{s}^{2}$ in Fig. 6(a). In short, the maximum throughput is approximately proportional to the square root of the maximum acceleration of vehicles, and we can increase the throughput by increasing the acceleration.
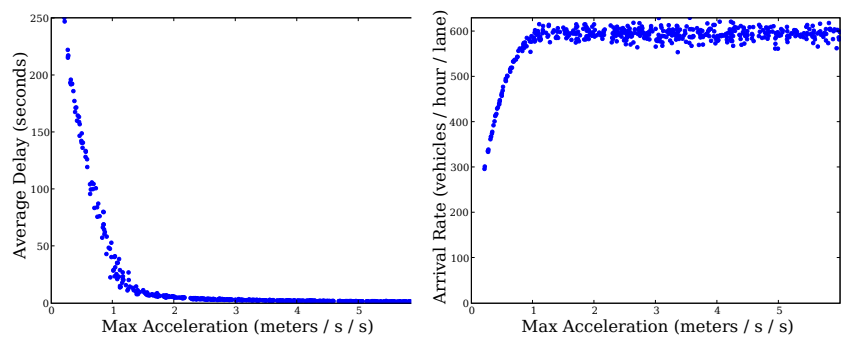

Fig. 6. Maximum acceleration versus average delay and arrival rate.

\section{Preventing Vehicles from Canceling their Reservations}

One of the keys to entering an intersection at a high speed is to not stop before the intersection. FCFS, by itself, reduces the number of vehicles that stop at an intersection, which is one of the main reasons FCFS is more efficient than traffic signals and stop signs [5]. While FCFS has done a good job in this regard, there is still room for improvement on the autonomous vehicles' side: the driver agents can also help prevent themselves from stopping before an intersection by requesting reservations that they can keep.

There are two scenarios in which a vehicle has to stop before an intersection in FCFS. First, the vehicle cannot obtain a reservation from the intersection manager and is forced to stop before an intersection. This happens when the traffic level is heavy and most of the future reservation tiles have been reserved by other vehicles in the system. Second, the vehicle successfully obtains a reservation but later determines that it will not arrive at the intersection at the time and/or velocity specified in the reservation. In this scenario the vehicle has to cancel the reservation and those reservation tiles may be wasted if they cannot be reassigned.

Poor estimation of arrival times and arrival velocities can lead to the cancellation of reservations. In previous work, the estimation of arrival times and arrival velocities is based on a heuristic we called the optimistic/pessimistic heuristic, that derives the arrival time and arrival velocity based on a prediction about whether the vehicle can arrive at the intersection without the intervention of other vehicles [8]. However, this heuristic does not guarantee that the vehicle can arrive at the intersection at the estimated arrival time or the estimated arrival velocity; in fact, our experiments showed that vehicles were often unable to reach the intersection at the correct time, forcing them to cancel their reservations after holding the reservations for quite some time.

To avoid this problem we propose a new approach to estimating the arrival time and arrival velocity. In our approach when a driver agent estimates its arrival time and arrival velocity, it also generates a sequence of control signals. These control signals, if followed correctly, ensure that the vehicle will arrive at the estimated arrival time and at the estimated arrival velocity. We formulate this estimation problem as the following multiobjective optimization problem: among all possible sequences of control signals that would allow the vehicle to enter an intersection, find one such that the arrival time is the smallest and the arrival velocity is the largest.

For an acceleration-based controller, the sequence of control signals is a time sequence of accelerations stating the acceleration the vehicle should take at every time step. We call a time sequence of accelerations an acceleration schedule. Like many multiobjective optimization problems, there is no single solution that dominates all other solutions in terms of both arrival time and arrival velocity. Here we choose arrival velocity as the primary objective, based on the analysis in Section IV-B. Our optimization procedure involves two steps: first, determine the highest possible arrival velocity the vehicle can achieve, and second, among all the acceleration schedules that yield the highest possible arrival velocity, find the one whose arrival time is the earliest.

We illustrate how the estimation procedure works using a time-velocity diagram as shown in Fig. 7. In this figure, $v_{1}$ is the current velocity of the vehicle, $t_{1}$ is the current time, $D$ is the distance between the current position of the vehicle and the intersection, $v^{\max }$ is the speed limit of the road, and $v_{2}^{\max }$ is the speed limit of the trajectory for the vehicle to safely traverse the intersection. Note that $v_{2}^{\max }$ is usually significantly smaller than $v^{\max }$ for turning trajectories so as to limit the centripetal force on the passengers. In addition, we define $a_{\max }$ and $a_{\min }$ to be the maximum acceleration and the maximum deceleration (minimum acceleration), respectively. We can see that any function $v(\cdot)$ in the time-velocity diagram that satisfies the following five constraints is a feasible velocity schedule for velocity-based controllers.

1) $v\left(t_{1}\right)=v_{1}$

2) $\int_{t_{1}}^{t_{\text {end }}} v(t) d t=D$, where $t_{\text {end }}$ is the arrival time (i.e., the distance traveled must be $D$ );

3) $v\left(t_{\text {end }}\right) \leq v_{2}^{\max }$ (i.e., the arrival velocity cannot exceed the speed limit of the trajectory);

4) $0 \leq v(t) \leq v^{\max }$ for $t_{1} \leq t \leq t_{\text {end }}$ (i.e., the velocity cannot exceed the speed limit of the road or be negative at any point in time); and

5) $a_{\text {min }} \leq \frac{d}{d t} v(t) \leq a_{\text {max }}$ for $0 \leq t \leq t_{\text {end }}$ (i.e., the acceleration at any point in time must be within the limitations).

We call $v(\cdot)$ a velocity schedule, which can be directly used by a velocity-based controller. A velocity schedule is feasible 
if it satisfies the above constraints. Our objective is to find a feasible velocity schedule $v(\cdot)$ such that $v\left(t_{\text {end }}\right)$ is as high as possible while $t_{\text {end }}$ is as small as possible. For accelerationbased controllers, we can compute the corresponding feasible acceleration schedule by the derivative of $v(\cdot)$ (i.e., $\frac{d}{d t} v(t)$ ). (a) Case 1: $\mathrm{Area}_{1}+\mathrm{Area}_{3} \leq D$

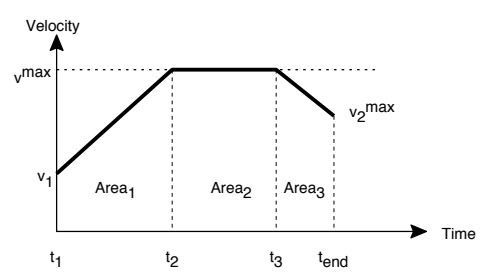

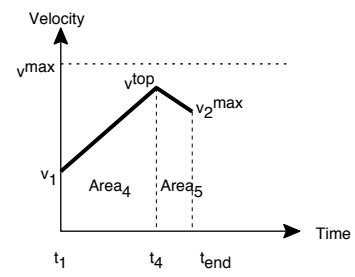

(b) Case 2: Area $1+\mathrm{Area}_{3}>D$
Fig. 7. The time-velocity diagrams for the estimation of the arrival time and the arrival velocity.

We propose an optimization procedure to find $v(\cdot)$ with the highest possible $v\left(t_{\text {end }}\right)$ and smallest $t_{\text {end }}$. First, compute the area of two trapezoids Area 1 and $\mathrm{Area}_{3}$ as shown in Fig. 7(a). To compute Area ${ }_{1}$, find a point $\left(t_{2}, v^{\max }\right)$ such that $\left(t_{2}, v^{\max }\right)$ is an interception of the line extending from $\left(t_{1}, v_{1}\right)$ with slope $a_{\max }$ and the horizontal line $v=v^{\max }$. We compute Area $a_{3}$ by choosing an arrival time $t_{\text {end }}^{\prime}$ and then find an intercepting point $\left(t_{3}^{\prime}, v^{\max }\right)$ between the line $v=v^{\max }$ and the line passing through the point $\left(t_{\text {end }}^{\prime}, v_{2}^{\max }\right)$ with slope $a_{\min }$.

If Area $_{1}+$ Area $_{3} \leq D$, the vehicle can accelerate to $v^{\max }$, maintain that speed for a certain period of time, then decelerate to $v_{2}^{\max }$, and finally reach the intersection (Case 1 in Fig. 7). Then Area $_{2}=D-$ Area $_{1}-$ Area $_{3}$ is nonnegative. Let $d$ be $\frac{\mathrm{Area}_{2}}{v^{\text {max }}}$. Then we can determine the actual value of $t_{3}^{\prime}$ and $t_{\text {end }}^{\prime}$ by $t_{3}=t_{2}+d$ and $t_{\text {end }}=t_{3}+\frac{2 \times \mathrm{Area}_{3}}{v^{\max }+v_{2}^{\max }}$. From this the optimization procedure can find a piecewise linear function for $v(\cdot)$ such that $v(\cdot)$ is a feasible velocity schedule. The optimization procedure returns the acceleration schedule $\left\langle\left(t_{1}, a_{\max }\right),\left(t_{2}, 0\right),\left(t_{3}, a_{\min }\right)\right\rangle$. If $\mathrm{Area}_{1}+\mathrm{Area}_{3}>D$, the vehicle cannot accelerate to $v^{\max }$ because $D$ is too small. But the vehicle can still accelerate to a velocity $v^{t o p}$ that is less than the speed limit $v^{\max }$ and immediately decelerate to $v_{2}^{\max }$ if $v^{t o p}$ exists (Case 2 in Fig. 7). However, if $v^{t o p}$ does not exist and $v_{1}>v_{2}^{\max }$, there is no feasible acceleration schedule since the vehicle is too close to the intersection.

We implemented the procedure and conducted an experiment to compare FCFS with traffic signals and stop signs using the realistic set of simulation parameters presented in Section III-B. Fig. 8 shows that even though the large buffer sizes and conservative vehicle parameters heavily reduce FCFS's performance, FCFS still does better than traffic signals and four-way stop signs.

\section{SUMMARY AND CONCLUSION}

In this paper we evaluated the AIM system in a mixed reality scenario, identified assumptions in the simulation that need relaxing, and determined FCFS parameters that enable an autonomous vehicle to safely traverse an intersection.

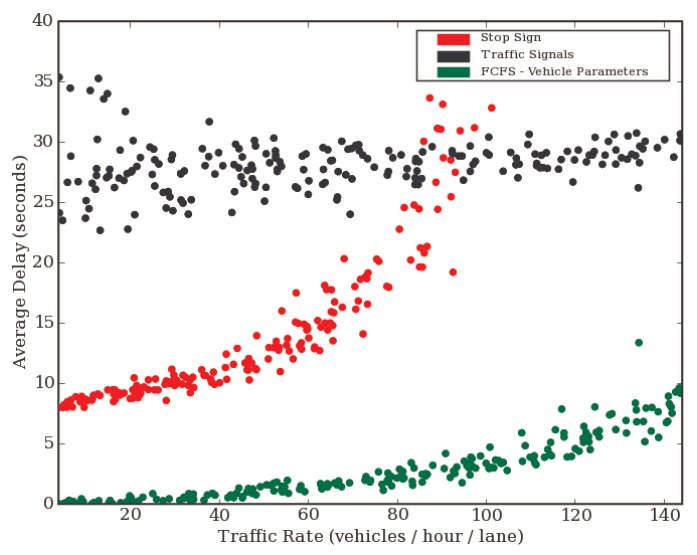

Fig. 8. Comparison of FCFS with traffic signals and four-way stop signs, using the larger buffer sizes and conservative vehicle parameters.

We explicated the relationship between buffer size and the throughput of an intersection via Little's Law, and proposed that increasing the vehicles acceleration and improving the accuracy of the estimation for the arrival time and arrival velocity will help throughput of the intersection. These findings allowed us to implement specific improvements to our autonomous vehicle with the result of achieving better, and fully realistic, FCFS performance. This research represents a vital step towards the longterm goal of validating and testing the AIM system with multiple fully autonomous cars in a dense urban setting.

\section{ACKNOWLEDGMENTS}

This work has taken place in the Learning Agents Research Group (LARG) at the Artificial Intelligence Laboratory, The University of Texas at Austin. LARG research is supported in part by grants from the National Science Foundation (CNS-0615104 and IIS-0917122), ONR (N00014-09-1-0658), DARPA (FA8650-08-C-7812), and the Federal Highway Administration (DTFH61-07-H-00030). Thanks also to Austin Robot Technology, and especially Jack O'Quinn, for their continuing contributions to the development and maintenance of Marvin.

\section{REFERENCES}

[1] R. Bishop, Intelligent Vehicle Technology and Trends. Artech House, 2005.

[2] R. Naumann, R. Rasche, and J. Tacken, "Managing autonomous vehicles at intersections," IEEE Intelligent Systems, vol. 13, no. 3, pp. 82-86, May 1998.

[3] USDOT, "Inside the USDOT's 'intelligent intersection' test facility," Newsletter of the ITS Cooperative Deployment Network, July 15, 2003 accessed online 17 May 2006 at http://www.ntoctalks.com/icdn/intell_ intersection.php.

[4] DARPA, "DARPA Urban Challenge," http://www.darpa.mil/ grandchallenge/index.asp, 2007.

[5] K. Dresner and P. Stone, "A multiagent approach to autonomous intersection management," Journal of Artificial Intelligence Research (JAIR), March 2008.

[6] P. Beeson, J. O'Quin, B. Gillan, T. Nimmagadda, M. Ristroph, D. Li, and P. Stone, "Multiagent interactions in urban driving," Journal of Physical Agents, vol. 2, no. 1, pp. 15-30, March 2008, special issue on Multi-Robot Systems.

[7] J. D. C. Little, "A Proof for the Queuing Formula: $L=\lambda W$," Operations Research, vol. 9, no. 3, pp. 383-387, 1961.

[8] K. Dresner, "Autonomous intersection management," Ph.D. dissertation, The University of Texas at Austin, 2009. 